\title{
Patterns of periodontal disease progression based on linear mixed models of clinical attachment loss
}

\author{
Ricardo Teles ${ }^{1,2}$ (1) | Kevin Moss ${ }^{3}$ | John S. Preisser ${ }^{4}$ | Robert Genco ${ }^{5}$ | \\ William V. Giannobile ${ }^{6}$ (D) | Patricia Corby ${ }^{7}$ | Nathalia Garcia ${ }^{8}$ | Heather Jared $^{9}$ | \\ Gay Torresyap $^{2}$ | Elida Salazar ${ }^{2}$ | Julie Moya ${ }^{7}$ | Cynthia Howard ${ }^{7}$ | Robert Schifferle ${ }^{5}$ | \\ Karen L. Falkner $^{5}$ | Jane Gillespie ${ }^{8}$ | Debra Dixon ${ }^{8}$ | MaryAnn Cugini ${ }^{2}$
}

${ }^{1}$ Department of Periodontics, Penn Dental Medicine, University of Pennsylvania,

Philadelphia, PA, USA

${ }^{2}$ Department of Applied Oral Sciences, Forsyth Institute, Cambridge, MA, USA

${ }^{3}$ Department of Dental Ecology, School of Dentistry, University of North Carolina at Chapel Hill, Chapel Hill, NC, USA

${ }^{4}$ Department of Biostatistics, Gillings School of Global Public Health, University of North Carolina at Chapel Hill, Chapel Hill, NC, USA

${ }^{5}$ Department of Oral Biology, School of Dental Medicine, State University of New York at Buffalo, Buffalo, NY, USA

${ }^{6}$ Department of Periodontics and Oral Medicine, Michigan Center for Oral Health Research, University of Michigan School of Dentistry, Ann Arbor, MI, USA

${ }^{7}$ New York University College of Dentistry, Bluestone Center for Clinical Research, New York, NY, USA

${ }^{8}$ Section of Periodontics, Department of Applied Dental Medicine, Southern Illinois University School of Dental Medicine, Alton, IL, USA

${ }^{9}$ Rho Inc., Contract Research Organization, Chapel Hill, NC, USA

\section{Correspondence}

Ricardo Teles, Department of Periodontics,

Penn Dental Medicine, University of

Pennsylvania, Philadelphia, PA, USA.

Email: rteles@upenn.edu

Funding information

This study was supported by research grant DE021127-01 from the National Institute of Dental and Craniofacial Research

\section{Abstract}

Aim: The goal of the present longitudinal cohort study was to examine patterns of periodontal disease progression at progressing sites and subjects defined based on linear mixed models (LMM) of clinical attachment loss (CAL).

Materials and Methods: A total of 113 periodontally healthy and 302 periodontitis subjects had their CAL calculated bimonthly for 12 months. LMMs were fitted for each site and the predicted CAL levels used to categorize their progression state. Participants were grouped based on the number of progressing sites into unchanged, transitional and active subjects. Patterns of periodontal disease progression were explored using descriptive statistics.

Results: Progression occurred primarily at molars (50\% of progressing sites) and interproximal sites (72\%), affected a higher proportion of deep than shallow sites $(2.7 \%$ versus $0.7 \%$ ), and pocketing was the main mode of progression (49\%). We found a low level of agreement (47\%) between the LMM and traditional approaches to determine progression such as change in $\mathrm{CAL} \geq 3 \mathrm{~mm}$. Fourteen per cent of subjects were classified as active and among those $93 \%$ had periodontitis. The annual mean rate of progression for the active subjects was $0.35 \mathrm{~mm} /$ year.

Conclusion: Progressing sites and subjects defined based on LMMs presented patterns of disease progression similar to those previously reported in the literature.

KEYWORDS

clinical attachment loss, disease progression, linear mixed models, periodontal disease 


\section{1 | INTRODUCTION}

Our understanding of periodontal disease progression comes from longitudinal studies examining the natural history of this condition. Longitudinal periodontal monitoring of untreated populations and subjects with access to different levels of oral care revealed common patterns of disease progression (Baelum, Luan, Chen, \& Fejerskov, 1997; Goodson, Tanner, Haffajee, Sornberger, \& Socransky, 1982; Haffajee \& Socransky, 1986; Heitz-Mayfield et al., 2003; Ismail, Morrison, Burt, Caffesse, \& Kavanagh, 1990; Lindhe, Haffajee, \& Socransky, 1983; Lindhe, Okamoto, Yoneyama, Haffajee, \& Socransky, 1989a; Loe, Anerud, Boysen, \& Morrison, 1986; Loe, Anerud, Boysen, $\&$ Smith, 1978a,b,c; Machtei et al., 1997; Millen et al., 2014; Schatzle et al., 2003; Socransky, Haffajee, Goodson, \& Lindhe, 1984). Although annual mean rates of progression varied greatly, the picture that emerges is of a slow progressing disease, with significant progression being a sporadic event and the majority of progressing sites concentrating on a relatively small number of subjects (Baelum et al., 1997; Lindhe et al., 1983; Schatzle et al., 2003). Although loss of attachment can affect any tooth and site in the mouth, periodontal disease progression seems to occur primarily in pre-molars and molars (Schatzle et al., 2003; Thomson, Broadbent, Poulton, \& Beck, 2006). Younger individuals seem to express loss of attachment through gingival recession, while pocketing becomes the main mode of disease progression as subjects get older (Schatzle et al., 2003). Pockets also seem to develop preferentially at interproximal sites, while the midbuccal and midlingual sites experience tissue destruction mainly through recession (Schatzle et al., 2003; Thomson et al., 2006). A tendency for the incidence of progression to increase with age has also been reported (Haffajee et al., 1991; Lindhe et al., 1989a), although this was not always the case (Baelum et al., 1997). Disease activity seems to affect a higher proportion of sites previously exposed to loss of attachment. However, because of the larger number of shallow sites in most subjects, progression affects a much higher number of sites without previous loss of attachment (Lindhe, Okamoto, Yoneyama, Haffajee, \& Socransky, 1989b; Thomson et al., 2006).

The vast majority of the literature reporting on periodontal disease progression has relied on pairs of visits to determine changes in clinical attachment loss (CAL). Disease progression was then defined based on increases in CAL above certain thresholds to account for "error" in these measurements (Aeppli, Boen, \& Bandt, 1985; Beck, Koch, \& Offenbacher, 1994; Deas, Pasquali, Yuan, \& Kornman, 1991; Goodson et al., 1982; Haffajee, Socransky, \& Goodson, 1983b; Jeffcoat \& Reddy, 1991; Lindhe et al., 1983). Even when measurements were obtained over several time points, reversals in CAL were, for the most, ignored (Lindhe et al., 1989a). Because of the uncertainties associated with a diagnosis of periodontal disease activity defined based on a single pair of measurements of CAL (Corraini, Baelum, \& Lopez, 2013; Tonetti \& Claffey, 2005), it is possible that the patterns of disease progression previously reported were somewhat inaccurate. We have recently reported on the use of linear mixed models (LMM) to classify periodontal sites according to changes in CAL over 12 months into different categories of

\section{Clinical Relevance}

Scientific rationale for the study: Studies exploring patterns of periodontal disease progression in the literature relied on a limited number of visits for monitoring and on pairs of clinical attachment loss (CAL) measurements to define progression. Principal findings: The use of linear mixed models had a poor agreement with traditional approaches to define disease progression using thresholds of changes in CAL calculated using a pair of visits. However, patterns of disease progression were quite similar to those previously reported in the literature.

Practical implications: Robust methods to define disease progression might result in more accurate assessments of the diagnostic and prognostic properties of clinical parameters and biomarkers.

disease progression (Teles et al., 2016). We proposed that the categories of periodontal disease progression based on LMMs of CAL overcome several of the limitations from previous methodologies. A higher level of accuracy in designating a site as having undergone disease progression is paramount for the description of patterns of periodontal disease progression.

The data presented here were obtained from an ongoing study to search for biomarkers of periodontal disease progression. As the previous report on the LMM approach to define disease progression, we have completed the clinical study, and 427 participants were monitored for disease progression for 12 months. The goal of the present report was to describe the patterns of periodontal disease progression in this population, using the LMM approach to identify progressing sites and subjects. This is the largest number of subjects ever followed for 12 months without treatment with bimonthly monitoring visits, allowing for a robust characterization of the patterns of periodontal disease progression.

\section{2 | MATERIALS AND METHODS}

\section{1 | Study design}

The data were obtained as part of a prospective multicentre clinical study on biomarkers of periodontal disease progression (Teles et al., 2016). Participants were recruited between January 2012 and December 2014 at four clinical centres in the United States: The Forsyth Institute (Cambridge, MA), New York University College of Dentistry (New York, NY), the University at Buffalo, State University of New York (Buffalo, NY) and Southern Illinois University School of Dental Medicine (Alton, IL). Calibrated clinicians examined participants bimonthly for 12 months to monitor changes in CAL measurements and determine disease progression. The Institutional Review Board from each centre approved the study prior to its initiation. 


\section{2 | Study population}

\subsection{1 | Inclusion and exclusion criteria}

The study had the following inclusion criteria: age $\geq 25$ years; willingness to comply with study protocol; $\geq 20$ natural teeth, excluding third molars; $\geq 12$ of these teeth had to be pre-molars, first or second molars. Periodontitis subjects were stratified into mild periodontal loss and severe periodontal loss. Participants with severe periodontal loss had at least eight separate teeth with at least one site of pocket depth $(\mathrm{PD}) \geq 5 \mathrm{~mm}$ and concomitant $\mathrm{CAL} \geq 3 \mathrm{~mm}$. Participants with mild periodontal loss had at least four teeth with at least one site of PD $\geq 5 \mathrm{~mm}$ and concomitant $\mathrm{CAL} \geq 2 \mathrm{~mm}$. Periodontitis subjects also had to present radiographic evidence of alveolar bone loss around at least two of the affected teeth. Periodontally healthy subjects met the following criteria: no radiographic evidence of alveolar bone loss, and all the teeth either had PD of $\leq 3 \mathrm{~mm}$, irrespective of the attachment level; $\mathrm{PD} \geq 4 \mathrm{~mm}$ with no CAL (except for the distal of the second molars); or, for distal of second molars, $\mathrm{PD}=4 \mathrm{~mm}$ with concomitant $\mathrm{CAL} \leq 2 \mathrm{~mm}$.

Exclusion criteria were as follows: presence of orthodontic appliances; presence of intra-oral lesions at the time of screening; gross tooth decay; root fragments, pericoronitis, endo-perio lesions or other dental abscesses; pregnancy or lactation; requirement for prophylactic antibiotics for dental procedures; periodontal or systemic antibiotic therapy in the previous 6 months; the use of tobacco products within 1 year before the screening visit; any medical condition that might influence the course of periodontal disease or treatment; chronic use of non-steroidal anti-inflammatory drugs; the use of chronic systemic immunosuppressive agents; hypersensitivity to tetracyclines; and participation in a clinical study within the last 30 days. Only participants who had attended both the baseline and 12-month monitoring visit and had the same examiner throughout the monitoring phase were included. Participants were allowed to have only one missing visit. Further details can be obtained at ClinicalTrials.gov (https://clinicaltrials.gov/ct2/home) under the identifier NCT01489839.

\subsection{2 | Clinical examination}

Participants had periodontal parameters measured at up to 168 sites per subject (six sites per tooth-mesiobuccal, buccal, distobuccal, mesiolingual, lingual and distolingual-for up to 28 teeth excluding third molars) including: probing depth (PD); measurement of distance from the cementoenamel junction (CEJ) to the free gingival margin ( $B$ measure) (in case of recession, a negative value was assigned); $C A L$ (calculated by subtracting the $B$ measure from the $P D$ ); presence or absence of plaque, gingival redness, BOP and suppuration. PD and the $\mathrm{B}$ measure were measured using calibrated North Carolina manual periodontal probes (PCPUNC $15 \mathrm{Hu}$-Friedy Co, Chicago, IL), rounding down to the nearest millimetre. At pre-molars, and at the first and second molars, PD and the B measure were measured twice. CAL was calculated for each pass by the electronic data capturing (EDC) system. If the difference between the two measurements was $\geq 2 \mathrm{~mm}$, the examiner was prompted by the EDC to obtain PD and the $\mathrm{B}$ measure a third time. The median CAL among the two or three passes was used for analysis.

\subsection{3 | Rescue therapy}

Subjects with $\geq 6$ sites with cumulative loss of attachment $\geq 2 \mathrm{~mm}$ from baseline during monitoring phase had their monitoring interrupted, and proceeded to treatment. Participants displaying $\geq 4 \mathrm{~mm}$ of CAL increase at a given site received periodontal rescue therapy at such sites and continued with monitoring. After the monitoring phase, periodontally healthy subjects received professional dental prophylaxis and exited the study, whereas participants with periodontal disease received non-surgical mechanical periodontal therapy.

\subsection{4 | Subjects and sites included in analyses}

All participants who completed the 12-month monitoring phase, attended at least six of seven monitoring visits, and were examined by the same examiner in all such visits, had their data analysed. Participants that had their monitoring interrupted due to rescue therapy were excluded. If a subject received rescue therapy in some but not all sites, data for such sites were removed from the analysis, and the subject was otherwise retained in the analysis for any remaining sites.

\section{3 | Linear mixed models}

We applied LMM to predict subject-specific trends in CAL for each site and from which classifications of progression and regression were made, as previously described (Teles et al., 2016). Briefly, for each of the 168 tooth sites, a separate linear mixed effects model with a cubic polynomial for time (months) was fitted to quantify the course of progression within individuals. For additional details on the LMM employed, refer to Teles et al., 2016 and the Supporting Information.

We then developed a threshold for progression empirically based on the prediction standard errors from a second series of LMM (again, one per site) fitted to $\triangle C A L_{i t}$, which is the change in $C A L$ value from baseline to time $=t$ (for $t=2,4,6,8,10$ or 12 months) for subject $i$. These models are identical to the models described above, except that the outcome is $\triangle C A L_{i j}$. The threshold for change was based on the 75th percentile of the distribution of the standard errors for subjectspecific predicted $\triangle C A L_{i j}$. Sites were then classified as progressing based on the predictions from the first series of LMM using the threshold established from the second series. We grouped sites based on changes in pCAL ( $\triangle \mathrm{pCAL}$ ) into: (i) regressing sites $\left(\triangle \mathrm{pCAL}<-2 \mathrm{Q}_{75}\right)$; (ii) stable sites $\left(-2 Q_{75} \leq \Delta \mathrm{pCAL} \leq 2 \mathrm{Q}_{75}\right)$; (iii) intermediate sites $\left(2 \mathrm{Q}_{75}<\Delta \mathrm{pCAL}<4 \mathrm{Q}_{75}\right)$; and (iv) progressing sites $\left(\Delta \mathrm{pCAL} \geq 4 \mathrm{Q}_{75}\right)$ (see Teles et al., 2016 for additional details).

\subsection{Data analyses}

All descriptive statistics, including mean periodontal clinical parameters and demographics, were computed using $S A S^{\circledR}$ software, and 
there was no imputation of missing data points. Mean periodontal clinical parameters were calculated for each subject and then across subjects in each clinical category separately for baseline and 12month data. The mean change in CAL from baseline to 12 months was computed to estimate the "annual mean rate of progression." Subjects were also grouped into categories of progression such as unchanged (0 progressing sites), transitional (1-2 progressing sites) and active ( $\geq 3$ progressing sites). Statistical significance of differences in the number of subjects in the three categories of progression across clinical groups (i.e., Healthy, Mild periodontal loss and Severe periodontal loss) was tested using the chi-square test. This analysis was also conducted grouping subjects based on CDC/AAP case definitions (Page \& Eke 2007; Eke et al. 2012). Mean clinical parameters were then calculated for subjects in the three progression categories for baseline and 12-month data, including the "annual mean rate of progression." Significance of statistical differences across progression categories was determined using ANOVA. For certain analyses, periodontally healthy subjects were excluded from the progression categories. The mode of disease progression (i.e., pocketing, recession or both) was determined based on changes in PD and B measure from baseline to 12 months.

\section{3 | RESULTS}

Of the 526 participants who attended a baseline visit, 53 subjects discontinued their participation after baseline due to different reasons, while 46 subjects had their monitoring interrupted due to rescue therapy (Figure 1). From the 427 participants who completed the 12-month monitoring, 12 were excluded because of a change in examiner or because they attended less than six monitoring visits, resulting in 415 participants in the final analysis. Among these participants, 62 sites were excluded due to rescue therapy for a final number of 66,193 sites included in analyses. Periodontally healthy subjects tended to be younger, more likely to be female, and to have fewer missing teeth than subjects with mild or severe periodontal loss (Table 1). One can also observe that subjects in the healthy category presented less plaque, gingival redness, BOP and suppuration than the periodontitis groups. However, subjects classified as "periodontally healthy" were not necessarily periodontally intact and had an average CAL of $1.1 \mathrm{~mm}$.

\section{1 | Safety summary}

During the study, there were no unanticipated problems or serious adverse events reported. Of 526 subjects who attended a baseline visit, 124 subjects required rescue therapy at the tooth site level but were able to remain in the monitoring phase of the study. Fifty-nine subjects transitioned to the treatment and maintenance phase to receive periodontal therapy based on the rescue therapy criteria for the study. Twenty-eight teeth had to be extracted during the monitoring phase of the study. Reasons for extractions included the following: pain (five teeth); dental caries (eight teeth); fracture (eight teeth); endodontic

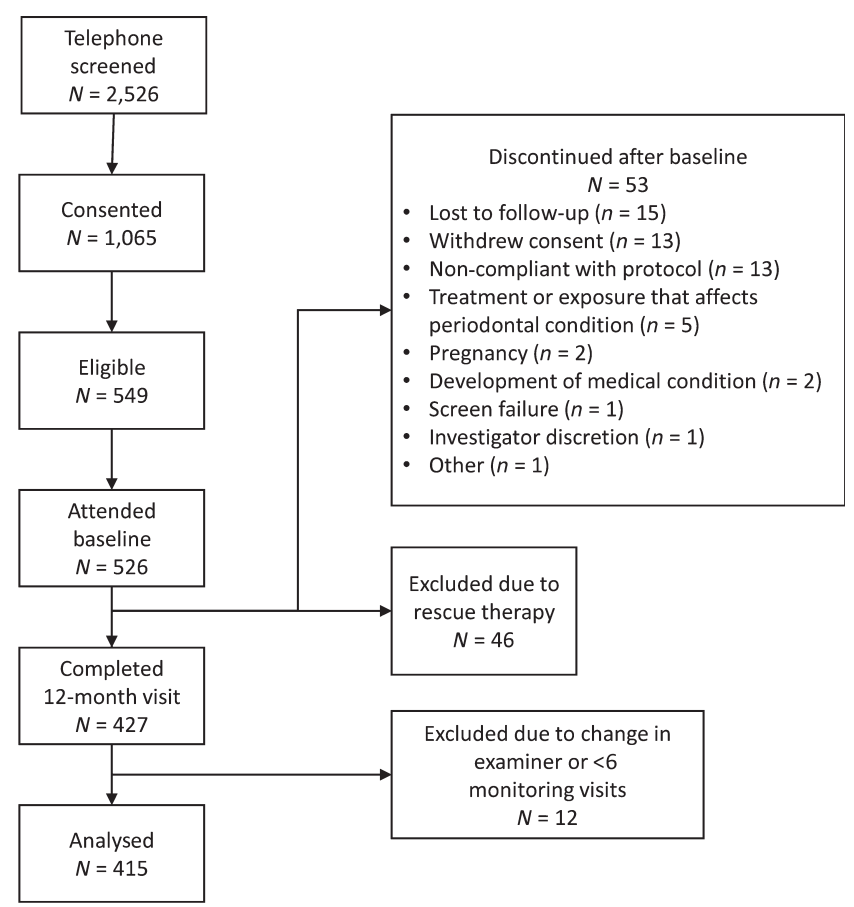

FIGURE 1 Flow chart of subject recruitment for the study: 2,526 subjects were telephone screened for this study; 1,065 subjects were enrolled (consented) in the study; 549 enrolled subjects were deemed eligible for the study after clinical screening; and 526 subjects attended a baseline visit. Of those, 53 discontinued due to different reasons, 46 subjects were moved to the treatment phase due to rescue therapy, and 427 subjects completed their 12-month visit. Twelve of these individuals were excluded due to change in the examiner during the monitoring phase, resulting in 415 subjects (113 periodontally healthy; 144 with mild periodontal loss and 158 with severe periodontal loss)

abscess (one tooth); third molar extraction (two teeth); periodontal reasons (two teeth); and undetermined causes (two teeth).

\subsection{LMM for changes in CAL measurements}

From the LMM for changes in CAL, the 75th percentile for the standard errors of prediction was 0.242 . Thus, the cut point for intermediate sites was selected as $0.474 \mathrm{~mm}$, while the cut point for progressing sites was set at $0.948 \mathrm{~mm}$.

Of 66,193 sites examined, $86.2 \%$ were classified as stable, and only $482(0.7 \%)$ were classified as progressing based on the results from the LMMs (Table 2). Subjects with severe periodontal loss had three times higher proportion of progressing sites compared to periodontally healthy subjects. Fifty-eight per cent of the regressing sites were present in the severe periodontal loss group. At the subject level, $44 \%$ of participants presented at least one progressing site. Among periodontally healthy subjects, $22 \%$ had at least one progressing site compared to $53 \%$ for periodontitis subjects.

By computing the proportions of progressing sites across different tooth positions in the arch, it became apparent that the majority of progressing sites were located in the posterior sextants (i.e., premolars and molars) (Figure 2). Regarding site positions around the 
TAB LE 1 Demographic and clinical parameters of study subjects in the three clinical categories: periodontally healthy subjects, subjects with mild periodontal loss and subjects with severe periodontal loss at baseline and 12 months

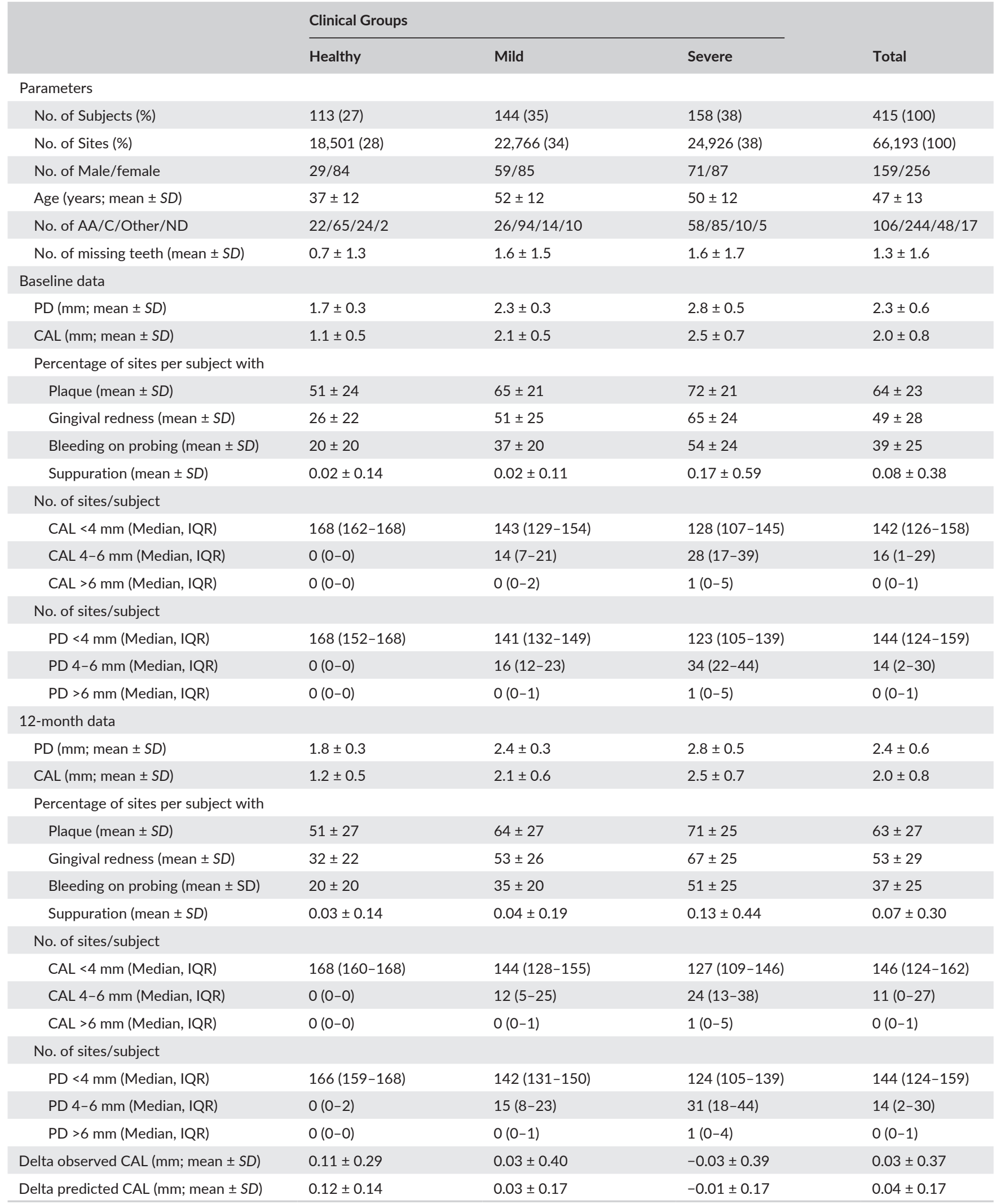

AA, African American; C, Caucasian; ND, Not disclosed; PD, probing depth; CAL, clinical attachment loss; IQR, inter-quartile range. 
TAB LE 2 Number and percentage of sites (in parenthesis) for each of the four categories of progression from baseline to month 12, with classifications based on linear mixed model predictions of CAL stratified in the three clinical groups

\begin{tabular}{|c|c|c|c|c|c|c|c|c|c|c|}
\hline \multirow{2}{*}{$\begin{array}{l}\text { Clinical Groups } \\
\text { Healthy }(N=113)\end{array}$} & \multicolumn{8}{|c|}{ Progression category } & & \\
\hline & 279 & $(0.4 \%)$ & 16,728 & (25.3\%) & 1,444 & $(2.2 \%)$ & 50 & (0.1\%) & 18,501 & (28.0\%) \\
\hline Mild PD $(N=144)$ & 1,393 & $(2.1 \%)$ & 19,592 & $(29.6 \%)$ & 1,580 & $(2.4 \%)$ & 214 & $(0.3 \%)$ & 22,779 & (34.4\%) \\
\hline $\begin{array}{r}\text { Severe PD } \\
(N=158)\end{array}$ & 2,315 & (3.5\%) & 20,717 & (31.3\%) & 1,663 & $(2.5 \%)$ & 218 & $(0.3 \%)$ & 24,913 & $(37.6 \%)$ \\
\hline
\end{tabular}

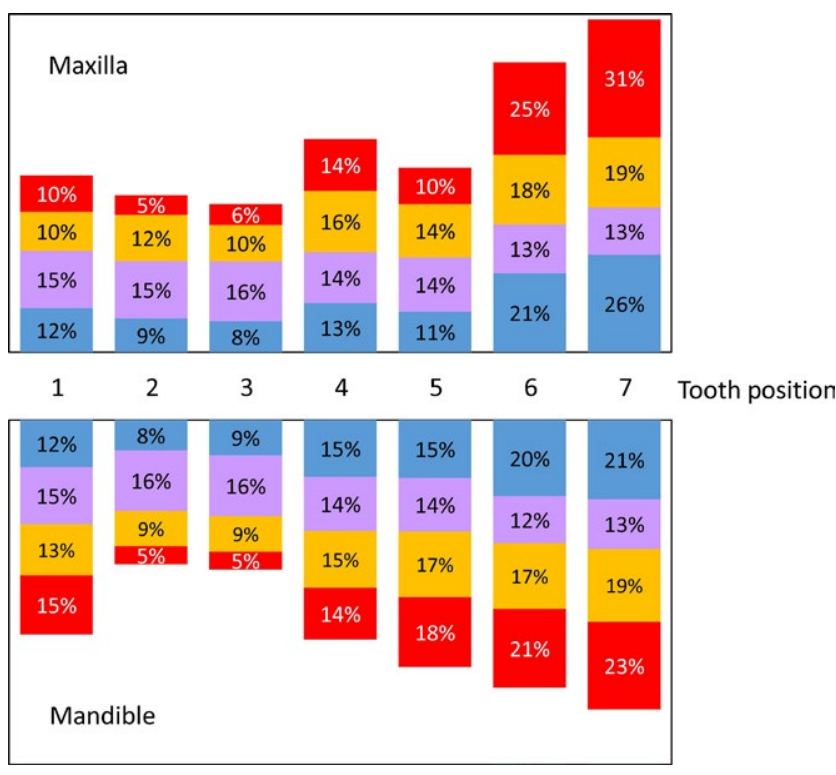

FIGURE 2 Stack bar graph of frequency of occurrence of sites in different categories of progression: regressing (blue bars), stable (purple bars), intermediate (orange bars) and progressing (red bars) at different tooth positions in the upper and lower arches. Numbers correspond to different teeth in a quadrant, based on the FDI World Dental Federation notation

tooth, progression occurred more often at the mesiobuccal and distobuccal sites and least often at the midbuccal site (Table 3). When the distribution of progressing sites across different baseline PDs was calculated, one could observe that, although deep sites had a higher proportion of progressing sites $(2.7 \%$ compared to $0.7 \%$ for shallow sites), progression affected a higher number of originally shallow sites (Table 4).

Pocketing was the main mechanism for disease progression, accounting for $48.7 \%$ of all progressing sites. Pure recession involved $21.7 \%$ of progressing sites, a combination of pocketing and recession affected $27.8 \%$, while $1.2 \%$ of the progressing sites did not change in either PD or CAL from baseline to 12 months. Overall, periodontally healthy subjects had a higher proportion of sites progressing through recession (32\%), compared to the mild periodontal loss (17\%) and severe periodontal loss (22\%) groups. When we examined the distribution of these three modes of loss of attachment across sites around the tooth, pure pocketing occurred mainly at interproximal sites. At the midbuccal site, disease progression occurred primarily through recession or a combination of pocketing and recession (Table S1).

We compared our categories of progression at the site level with traditional definitions of progression based on changes in $\mathrm{CAL}$ greater or equal to 2 or $3 \mathrm{~mm}$. Only 257 sites (0.4\%) had a change in CAL equal to or greater than $3 \mathrm{~mm}$ from baseline to 12 months, compared to 482 by LMM. Of those, only $47 \%$ were classified as progressing based on the LMM, $44 \%$ as intermediate and $8 \%$ as stable. One thousand eight hundred and ninety-six sites (2.9\%) had a CAL change greater than or equal to $2 \mathrm{~mm}$ over 12 months. Nineteen per cent of these sites were classified as progressing, 54\% as intermediate and $27 \%$ as stable.

We computed the distribution of participants into three categories of progression based on the number of progressing sites across clinical groups, defined based on our case definitions and the AAP/ CDC case definitions. Results indicated that there was an statistically significantly higher proportion of active subjects in the periodontitis groups compared to healthy subjects, irrespective of the case definition used (Table 5).

The three categories of progressing subjects had comparable mean age, demographic parameters and number of missing teeth at baseline (Table S2). However, there were statistically significant differences in most clinical parameters, with the exception of suppuration. When clinical parameters were compared across progression categories after the exclusion of periodontally healthy subjects, the majority of statistically significant differences disappeared (Table 5). However, the 12-month data revealed that most clinical parameters in the active group worsened during monitoring (Table 6). For instance, the change in mean number of sites with PD $\geq 4 \mathrm{~mm}$ was 5.4 for the active group compared to 0.1 and -6.6 for the transitional and unchanged groups, respectively. This resulted in statistically significant differences in most clinical parameters across categories of progression.

\section{4 | DISCUSSION}

The findings reported here were based on the largest cohort of subjects to be monitored for periodontal disease progression without therapy thus far. We used LMM of repeated measures of CAL to compensate 
TA B LE 3 Number and percentage of sites (in parenthesis) for each of the four categories of progression at each site position around the tooth

\begin{tabular}{|c|c|c|c|c|c|c|c|c|c|c|c|c|}
\hline \multirow[b]{2}{*}{ Progression category } & \multicolumn{12}{|c|}{ Site position } \\
\hline & MB & & B & & DB & & $\mathrm{DL}$ & & $\mathbf{L}$ & & $M L$ & \\
\hline Regression & 680 & (6\%) & 821 & $(7 \%)$ & 805 & $(7 \%)$ & 634 & $(6 \%)$ & 478 & $(4 \%)$ & 569 & $(5 \%)$ \\
\hline Stable & 9,282 & (84\%) & 9,458 & (86\%) & 9,310 & (84\%) & 9,592 & (87\%) & 9,876 & (89\%) & 9,519 & (86\%) \\
\hline Intermediate & 973 & $(9 \%)$ & 704 & $(6 \%)$ & 819 & $(7 \%)$ & 712 & $(6 \%)$ & 616 & $(6 \%)$ & 863 & $(8 \%)$ \\
\hline Progression & 102 & $(0.9 \%)$ & 64 & $(0.6 \%)$ & 97 & (0.9\%) & 77 & (0.7\%) & 68 & $(0.6 \%)$ & 74 & $(0.7 \%)$ \\
\hline
\end{tabular}

MB, mesiobuccal; B, midbuccal; DB, distobuccal; DL, distolingual; L, midlingual; ML, mesiolingual.

TABLE 4 Number and percentage of sites (in parenthesis) for each of the four categories of progression stratified according to baseline pocket depth category

\begin{tabular}{llrrr}
\multicolumn{5}{c}{ Progression category } \\
\cline { 2 - 5 } Baseline PD & Regressing & \multicolumn{1}{l}{ Stable } & Intermediate & Progressing \\
\hline $44 \mathrm{~mm}$ & $2,359(4 \%)$ & $50,701(88 \%)$ & $4,160(7.2 \%)$ & $387(0.7 \%)$ \\
\hline $4-6 \mathrm{~mm}$ & $1,447(18 \%)$ & $6,010(75 \%)$ & $492(6 \%)$ & $80(1.0 \%)$ \\
$>6 \mathrm{~mm}$ & $181(33 \%)$ & $326(58 \%)$ & $35(6 \%)$ & $15(2.7 \%)$ \\
\hline
\end{tabular}

TAB LE 5 Number and percentage of subjects (in parenthesis) for each of the three categories of progression stratified according to clinical groups based of ours and the CDC/AAP case definitions

\begin{tabular}{|ccccccc|} 
& \multicolumn{2}{c}{ Unchanged } & Transitional & \multicolumn{2}{c|}{ Active* $^{*}$} \\
\hline PD CLASS & & & & & & \\
\hline Healthy & 88 & $(78 \%)$ & 17 & $(15 \%)$ & 8 & $(7 \%)$ \\
\hline Mild & 69 & $(48 \%)$ & 47 & $(33 \%)$ & 28 & $(19 \%)$ \\
\hline Severe & 74 & $(47 \%)$ & 48 & $(30 \%)$ & 36 & $(23 \%)$ \\
\hline CDC/AAP & & & & & & \\
\hline Healthy & 96 & $(77 \%)$ & 18 & $(14 \%)$ & 11 & $(9 \%)$ \\
\hline $\begin{array}{l}\text { Mild/moderate } \\
\text { Severe }\end{array}$ & 84 & $(53 \%)$ & 51 & $(32 \%)$ & 24 & $(15 \%)$ \\
\hline
\end{tabular}

${ }^{*} p<.0001$ based on chi-square test.

for the high variability in this clinical parameter and achieve a more accurate diagnosis of disease progression (Teles et al., 2016). In the current study, we demonstrated that certain patterns of periodontal disease progression previously reported were also identifiable using this novel approach. For instance, advanced progression concentrated in a relatively small number of subjects, progression was a rare event, progression occurred primarily at interproximal sites, progression affected mainly molars, progression at interproximal surfaces occurred mainly through pocketing, while midbuccal sites had a higher prevalence of recession, and sites with deeper pockets tended to display a higher proportion of disease progression (Albandar, Rise, Gjermo, \& Johansen, 1986; Baelum et al., 1997; Haffajee, Socransky, \& Goodson, 1983a; Ismail et al., 1990; Lindhe et al., 1989a; Loe et al., 1986; Papapanou, Wennstrom, \& Grondahl, 1989; Schatzle et al., 2003).

Although the prevalence of progression was higher at sites with baseline PD $>6 \mathrm{~mm}(2.7 \%)$ than at sites with PD $<4 \mathrm{~mm}(0.7 \%)$, the number of shallow sites progressing $(N=387)$ was much higher than for deeper sites ( $N=15$ ), a finding in accord with others (Lindhe et al., 1989b; Thomson et al., 2006). This observation reaffirms the need for clinicians to focus of the disease process that afflicts their patients, rather than on the sequelae of this process (i.e., deep pockets). The burden in terms of treatment needs imposed by disease progression is primarily the consequence of new diseased sites, rather than progression on previously affected sites.

Despite our best efforts to address hopeless teeth prior to enrolment, and a series of safety rules that triggered rescue therapy of monitored teeth and/or subjects, 28 teeth were lost during the monitoring phase of the study. In addition, $24 \%$ of participants required rescue therapy due to having at least one site with an increase in CAL greater than $3 \mathrm{~mm}$. Most extractions were associated with dental caries, fracture or pain associated with pulp pathology, and only two teeth were extracted due to deterioration of the periodontal condition. Although the number of teeth lost during the study was greater than desirable, the mean annual incidence of tooth loss per periodontitis subject (0.08/year) was similar to reports in the literature for subjects under periodontal maintenance (Checchi, Montevecchi, Gatto, \& Trombelli, 2002; Teles, Patel, Socransky, \& Haffajee, 2008; Wood, Greco, \& McFall, 1989). That indicates that the tooth loss observed in our population of untreated subjects, compared favourably to studies on periodontitis patients receiving maintenance. This suggests that despite the lack of therapy, our participants were not exposed to an undue risk for tooth loss.

We calculated CAL based on measurements of PD and the distance from the cementoenamel junction (CEJ) to the free gingival margin. Although this method has been employed extensively in the periodontal literature, including large epidemiological surveys such as the National Health and Nutrition Examination Survey (NHANES) (Eke et al., 2015), some have argued that indirect measures of CAL can add to error because it requires two separate measurements (Corraini et al., 2013). However, direct measures of clinical CAL 
TAB LE 6 Clinical parameters of periodontitis subjects in the three categories of progression: unchanged (no progressing sites), transitional (subjects with 1-2 progressing sites), and active (subjects with three or more progressing sites) at baseline and 12 months

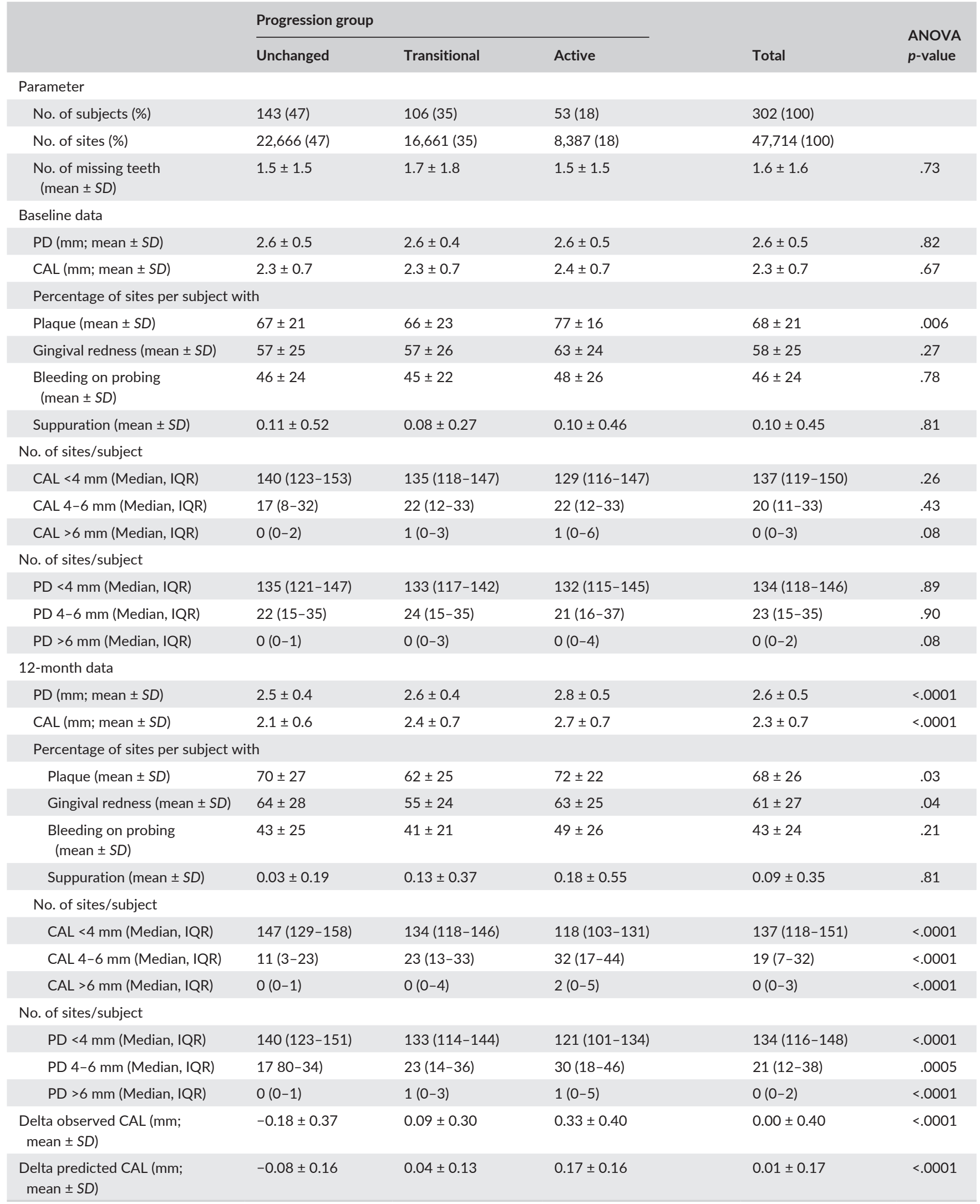

$\mathrm{PD}$, probing depth; CAL, clinical attachment loss; IQR, inter-quartile range. 
require considerable mental effort during the examination of sites where the CEJ is not exposed. For those areas, the examiner must mentally subtract the measured distance from the free gingival margin to the CEJ from the PD (Corraini et al., 2013). In our experience, this method is not without inaccuracies and slows down the examination considerably. Further, the reproducibility obtained in CAL measures in our study compares favourably with the published literature, as can be ascertained by the high percentage of agreement and small standard deviations achieved with the two passes (Supporting Information).

We arbitrarily grouped our subjects based on the number of progressing sites into "unchanged," "transitional" and "active" subjects. By comparing the annual mean rate of progression for the subjects classified as active with historical controls (Baelum et al., 1997; Ismail et al., 1990; Lindhe et al., 1989a; Machtei et al., 1997; Schatzle et al., 2001, 2003), one can observe that we were able to select subjects with a high annual mean rate of progression. In the classic report on the natural history of periodontal disease in man by Loe et al. (1986), the individuals identified as having rapid progression of periodontal disease had a mean annual rate varying from 0.10 to $1.0 \mathrm{~mm} /$ year. Among our subgroup of active participants, the range was -0.66 to $1.2 \mathrm{~mm} /$ year, and the mean was $0.35 \mathrm{~mm} /$ year. We interpreted these findings as indicative of the robustness of our criteria for selecting progressing sites and subjects.

We grouped subjects on three clinical groups based on their periodontal status. These criteria were established to accept a certain level of CAL as compatible with periodontal health, and to secure the recruitment of periodontitis subjects with a more severe and generalized pattern of disease, increasing our chances of observing disease progression. The drawback of establishing our own categories of disease was that the data cannot be promptly related to well-established standard case definitions, such as those proposed by the CDC and AAP (Page \& Eke 2007; Eke et al. 2012). To partially address this concern, we determined the prevalence of unchanged, transitional and active subjects in both classifications. The results were reassuring given that the proportions of subjects in the three categories of progression were similar in each clinical group across the two classifications. For instance, the proportion of active subjects in the severe group was $28 \%$ and $23 \%$ for the CDC/AAP classification and our case definitions, respectively. These findings support the notion that our disease categories behaved similarly to those define based on CDC/AAP criteria, regarding susceptibility to progression.

When mean demographic and clinical parameters were compared across subject-level categories of progression, we observed that even at baseline, active subjects presented worse periodontal parameters. This would suggest that participants with more severe periodontal disease were more prone to progression. However, when only periodontitis subjects were examined, there were only minor differences across categories of progression at baseline. Due to a worsening of clinical parameters in the active group and an improvement in the unchanged group, by 12 months, differences in clinical parameters were statistically significant across progressing groups. These findings support the notion that our definition of active subjects was capable of identifying individuals whose periodontal condition deteriorated during the one year of monitoring.

Previous studies on the progression of periodontal diseases reported pocketing as the primary mode of progression for individuals in the $45-49$ age range; accounting for $73.3 \%$ of progressing sites, while pure recession and a combination of recession and pocketing affected $21.7 \%$ and $5.0 \%$ of progressing sites, respectively (Schatzle et al., 2003). In our study population, $48.7 \%$ of sites progressed through pocketing, $21.7 \%$ through recession and $27.8 \%$ progressed through a combination of both mechanisms. Direct comparisons between our study and previous ones are compromised by the uniqueness of our approach to identify progressing sites using LMMs. Previous work has relied primarily on changes in CAL measurements obtained in a pair of visits to define progression. However, our results are in accord with the notion that pocketing is the primary mode of periodontal disease progression in adults. Further, our results confirmed previous studies indicating that buccal sites progress mainly through recession, while interproximal sites deteriorate primarily through pocketing (Schatzle et al., 2003). Also in accord with previous literature, periodontally healthy subjects had a higher proportion of sites progressing through recession compared with periodontitis individuals (Schatzle et al., 2003).

An important methodological difference between our study and the one by Schatzle et al. (2003) is that they examined data from two sites (mesial, buccal) per tooth in the beginning and four sites (mesial, buccal distal, lingual, i.e., 50\% buccal/lingual, 50\% interproximal) later, while we measured six sites per tooth (33\% buccal/lingual, $67 \%$ interproximal). Because interproximal sites progress mainly by pocketing, this difference in methodology may have impacted differences in the findings from the two studies.

Determining the mode of progression for progressing sites defined using LMMs also revealed certain limitations of this approach and a small percentage of progressing sites (1.7\%) did not demonstrate increases in either PD or recession. Close inspection of the longitudinal profiles of observed and predicted CAL for these eight sites, revealed that outliers of CAL measurements resulted in the upward trend of change in the predicted CAL values. Another pattern observed suggested reversal of transitory changes in CAL measurements (data not shown).

In a previous paper, we illustrated how the LMM approach selected progression sites with a lower tendency to have increases in CAL reverse in subsequent visits, compared to sites selected based on changes in CAL on a pair of visits (Teles et al., 2016). When comparing the number of sites classified as progressing using the LMM to those using thresholds of changes in CAL measurements, for the higher threshold of $\geq 3 \mathrm{~mm}$, only $47 \%$ of sites were classified as progressing based on the LMM. The lower threshold of $2 \mathrm{~mm}$ to define progression resulted in even higher proportions of mismatches to the LMM categories of progression. As highlighted in our previous paper on the LMM approach, thus far, we have no means to determine with certainty which sites truly underwent progression. However, these results illustrate the discrepancies that would result from selecting different methods to define periodontal disease progression. 
In summary, the use of LMM to characterize sites and subjects undergoing periodontal disease progression resulted in patterns of progression similar to those previously described in the literature. However, comparisons to traditional means to determine periodontal disease progression resulted in considerable discrepancies between which sites would be defined as progressing. Improvements in the diagnosis of sites and subjects that experienced periodontal disease progression might lead to more accurate assessments of the diagnostic and prognostic properties of clinical parameters and biomarkers. Future approaches using real-time assessment of disease activity based on biomarkers may improve the measurement accuracy of periodontal disease progression in at-risk patients.

\section{CONFLICT OF INTEREST}

The authors have stated explicitly that there are no conflicts of interest in connection with this article.

\section{ORCID}

Ricardo Teles (iD http://orcid.org/0000-0002-4216-2812

William V. Giannobile iD http://orcid.org/0000-0002-7102-9746

\section{REFERENCES}

Aeppli, D. M., Boen, J. R., \& Bandt, C. L. (1985). Measuring and interpreting increases in probing depth and attachment loss. Journal of Periodontology, 56, 262-264. https://doi.org/10.1902/jop.1985.56.5.262

Albandar, J. M., Rise, J., Gjermo, P., \& Johansen, J. R. (1986). Radiographic quantification of alveolar bone level changes. A 2-year longitudinal study in man. Journal of Clinical Periodontology, 13, 195-200.

Baelum, V., Luan, W. M., Chen, X., \& Fejerskov, O. (1997). A 10-year study of the progression of destructive periodontal disease in adult and elderly Chinese. Journal of Periodontology, 68, 1033-1042. https://doi. org/10.1902/jop.1997.68.11.1033

Beck, J. D., Koch, G. G., \& Offenbacher, S. (1994). Attachment loss trends over 3 years in community-dwelling older adults. Journal of Periodontology, 65, 737-743. https://doi.org/10.1902/jop.1994.65.8.737

Checchi, L., Montevecchi, M., Gatto, M. R., \& Trombelli, L. (2002). Retrospective study of tooth loss in 92 treated periodontal patients. Journal of Clinical Periodontology, 29, 651-656.

Corraini, P., Baelum, V., \& Lopez, R. (2013). Reliability of direct and indirect clinical attachment level measurements. Journal of Clinical Periodontology, 40, 896-905. https://doi.org/10.1111/jcpe.12137

Deas, D. E., Pasquali, L. A., Yuan, C. H., \& Kornman, K. S. (1991). The relationship between probing attachment loss and computerized radiographic analysis in monitoring progression of periodontitis. Journal of Periodontology, 62, 135-141. https://doi.org/10.1902/ jop.1991.62.2.135

Eke, P. I., Dye, B. A., Wei, L., Slade, G. D., Thornton-Evans, G. O., Borgnakke, W. S., ... Genco, R. J. (2015). Update on prevalence of periodontitis in adults in the United States: NHANES 2009 to 2012. Journal of Periodontology, 86, 611-622. https://doi.org/10.1902/ jop.2015.140520

Goodson, J. M., Tanner, A. C., Haffajee, A. D., Sornberger, G. C., \& Socransky, S. S. (1982). Patterns of progression and regression of advanced destructive periodontal disease. Journal of Clinical Periodontology, 9, 472-481.

Haffajee, A. D., \& Socransky, S. S. (1986). Attachment level changes in destructive periodontal diseases. Journal of Clinical Periodontology, 13, 461-475.
Haffajee, A. D., Socransky, S. S., \& Goodson, J. M. (1983a). Clinical parameters as predictors of destructive periodontal disease activity. Journal of Clinical Periodontology, 10, 257-265.

Haffajee, A. D., Socransky, S. S., \& Goodson, J. M. (1983b). Comparison of different data analyses for detecting changes in attachment level. Journal of Clinical Periodontology, 10, 298-310.

Haffajee, A. D., Socransky, S. S., Lindhe, J., Kent, R. L., Okamoto, H., \& Yoneyama, T. (1991). Clinical risk indicators for periodontal attachment loss. Journal of Clinical Periodontology, 18, 117-125.

Heitz-Mayfield, L. J., Schatzle, M., Loe, H., Burgin, W., Anerud, A., Boysen, H., \& Lang, N. P. (2003). Clinical course of chronic periodontitis. II. Incidence, characteristics and time of occurrence of the initial periodontal lesion. Journal of Clinical Periodontology, 30, 902-908.

Ismail, A. I., Morrison, E. C., Burt, B. A., Caffesse, R. G., \& Kavanagh, M. T. (1990). Natural history of periodontal disease in adults: Findings from the Tecumseh Periodontal Disease Study, 1959-87. Journal of Dental Research, 69, 430-435.

Jeffcoat, M. K., \& Reddy, M. S. (1991). Progression of probing attachment loss in adult periodontitis. Journal of Periodontology, 62, 185-189.

Lindhe, J., Haffajee, A. D., \& Socransky, S. S. (1983). Progression of periodontal disease in adult subjects in the absence of periodontal therapy. Journal of Clinical Periodontology, 10, 433-442.

Lindhe, J., Okamoto, H., Yoneyama, T., Haffajee, A., \& Socransky, S. S. (1989a). Longitudinal changes in periodontal disease in untreated subjects. Journal of Clinical Periodontology, 16, 662-670.

Lindhe, J., Okamoto, H., Yoneyama, T., Haffajee, A., \& Socransky, S. S. (1989b). Periodontal loser sites in untreated adult subjects. Journal of Clinical Periodontology, 16, 671-678.

Loe, H., Anerud, A., Boysen, H., \& Morrison, E. (1986). Natural history of periodontal disease in man. Rapid, moderate and no loss of attachment in Sri Lankan laborers 14 to 46 years of age. Journal of Clinical Periodontology, 13, 431-445.

Loe, H., Anerud, A., Boysen, H., \& Smith, M. (1978a). The natural history of periodontal disease in man. The rate of periodontal destruction before 40 years of age. Journal of Periodontology, 49, 607-620. https://doi. org/10.1902/jop.1978.49.12.607

Loe, H., Anerud, A., Boysen, H., \& Smith, M. (1978b). The natural history of periodontal disease in man. Study design and baseline data. Journal of Periodontal Research, 13, 550-562.

Loe, H., Anerud, A., Boysen, H., \& Smith, M. (1978c). The natural history of periodontal disease in man. Tooth mortality rates before 40 years of age. Journal of Periodontal Research, 13, 563-572.

Machtei, E. E., Dunford, R., Hausmann, E., Grossi, S. G., Powell, J., Cummins, D., ... Genco, R. J. (1997). Longitudinal study of prognostic factors in established periodontitis patients. Journal of Clinical Periodontology, 24, 102-109.

Millen, A. E., Andrews, C. A., LaMonte, M. J., Hovey, K. M., Swanson, M., Genco, R. J., \& Wactawski-Wende, J. (2014). Vitamin D status and 5year changes in periodontal disease measures among postmenopausal women: The Buffalo OsteoPerio Study. Journal of Periodontology, 85, 1321-1332. https://doi.org/10.1902/jop.2014.130686

Papapanou, P. N., Wennstrom, J. L., \& Grondahl, K. (1989). A 10-year retrospective study of periodontal disease progression. Journal of Clinical Periodontology, 16, 403-411.

Schatzle, M., Land, N. P., Anerud, A., Boysen, H., Burgin, W., \& Loe, $H$. (2001). The influence of margins of restorations of the periodontal tissues over 26 years. Journal of Clinical Periodontology, 28, 57-64.

Schatzle, M., Loe, H., Lang, N. P., Heitz-Mayfield, L. J., Burgin, W., Anerud, A., \& Boysen, H. (2003). Clinical course of chronic periodontitis. III. Patterns, variations and risks of attachment loss. Journal of Clinical Periodontology, 30, 909-918.

Socransky, S. S., Haffajee, A. D., Goodson, J. M., \& Lindhe, J. (1984). New concepts of destructive periodontal disease. Journal of Clinical Periodontology, 11, 21-32. 
Teles, R., Benecha, H. K., Preisser, J. S., Moss, K., Starr, J. R., Corby, P., ... Cugini, M. (2016). Modelling changes in clinical attachment loss to classify periodontal disease progression. Journal of Clinical Periodontology, 43, 426-434. https://doi.org/10.1111/jcpe.12539

Teles, R. P., Patel, M., Socransky, S. S., \& Haffajee, A. D. (2008). Disease progression in periodontally healthy and maintenance subjects. Journal of Periodontology, 79, 784-794. https://doi.org/10.1902/ jop.2008.070485

Thomson, W. M., Broadbent, J. M., Poulton, R., \& Beck, J. D. (2006). Changes in periodontal disease experience from 26 to 32 years of age in a birth cohort. Journal of Periodontology, 77, 947-954. https://doi. org/10.1902/jop.2006.050319

Tonetti, M. S., \& Claffey, N. (2005). Advances in the progression of periodontitis and proposal of definitions of a periodontitis case and disease progression for use in risk factor research. Group C consensus report of the 5th European Workshop in Periodontology. Journal of Clinical Periodontology, 32(Suppl 6), 210-213. https://doi. org/10.1111/j.1600-051X.2005.00822.x
Wood, W. R., Greco, G. W., \& McFall, W. T. Jr (1989). Tooth loss in patients with moderate periodontitis after treatment and long-term maintenance care. Journal of Periodontology, 60, 516-520. https://doi. org/10.1902/jop.1989.60.9.516

\section{SUPPORTING INFORMATION}

Additional Supporting Information may be found online in the supporting information tab for this article.

How to cite this article: Teles R, Moss K, Preisser JS, et al. Patterns of periodontal disease progression based on linear mixed models of clinical attachment loss. J Clin Periodontol. 2018;45:15-25. https://doi.org/10.1111/jcpe.12827 